\title{
Femtosecond energy transfer between chromophores in allophycocyanin trimers
}

\author{
A.V. Sharkov ${ }^{\text {a }}$, I.V. Kryukov ${ }^{\text {a }}$, E.V. Khoroshilov ${ }^{\text {a }}$, P.G. Kryukov ${ }^{a}$, R. Fischer ${ }^{b}$, \\ H. Scheer ${ }^{b}$ and T. Gillbro ${ }^{c}$ \\ a.N. Lebedev Physics Institute, Russian Academy of Sciences, 117924 Moscow, Russia \\ b Botanisches Institut der Universität, Menzinger Strasse 67, W-8000 Munich 19, Germany \\ c Department of Physical Chemistry, University of Umeà, S-901 87 Umeà, Sweden
}

Received 20 October 1991; in final form 13 February 1992

\begin{abstract}
Ultrafast energy-transfer processes in allophycocyanin (APC) trimers from Mastigocladus laminosus have been examined by a femtosecond absorption technique. Isotropic absorption recovery kinetics with $\tau=440 \pm 30 \mathrm{fs}$ were observed in APC trimers at $615 \mathrm{~nm}$. In APC monomers such a fast process was not observed. The anisotropy in both samples was constant and close to 0.4 during the first few picoseconds. The results are consistent with a model of the APC trimer in which the two APC chromophores have different absorption spectra with maxima about 600 and $650 \mathrm{~nm}$. The transfer of energy from the $600 \mathrm{~nm}$ chromophore to the $650 \mathrm{~nm}$ chromophore occurs in $440 \mathrm{fs}$ and is dominated by the Förster dipole-dipole energy-transfer mechanism.
\end{abstract}

\section{Introduction}

Our knowledge about energy transfer in the phycobilisome antenna complexes of blue-green bacteria and red algae has increased substantially during the last decade [ 1,2$]$. This is especially true for the overall energy transfer between the different pigment pools, i.e. between phycocyanin and allophycocyanin and from this pool to the chlorophyll a antenna of photosystem II in the thylakoid membrane [3,4]. Important questions under investigation that still remain open are related to the transfer of excitation energy between nearby chromophores in aggregates of C-phycocyanin (C-PC), allophycocyanin (APC), etc. For instance, there are different models for the energy transfer. Some workers support an exciton mechanism [5] while others prefer Förster-type transfer [6]. Of special interest is the allophycocyanin trimer. The monomeric unit contains two kinds of chromophores. They have the same molecular structure, viz. phycocyanobilin, but differ in their protein environments. One is bound to cystein $\alpha-80$, one to cysteins $\beta-81$ (species specific absolute numbering) [7]. The optical spectrum of the monomer $(\alpha \beta)_{1}$ shows a broad band with a maximum at 615 $\mathrm{nm}$ [1]. In contrast to this, the spectrum of the trimer, $(\alpha \beta)_{3}$, has a sharp peak at $653 \mathrm{~nm}$ and a shoulder at about $600 \mathrm{~nm}$. The reason for this spectral shift is not clear, but based on CD spectra it has been suggested that a strong excitonic interaction between the two chromophores might be the main cause of the shift $[8,9]$. An alternative explanation is that a conformational change occurs in at least one of the chromophores upon aggregation, causing the red-shift of the absorption maximum and giving the trimer a different $C D$ spectrum from the monomer.

In this Letter, we have resolved the ultrafast energy transfer between the $\alpha-80$ and $\beta-81$ chromophores and found it to be $440 \pm 30$ fs. Results from time-resolved anisotropy measurements at $615 \mathrm{~nm}$ show that the fast process is probably not due to relaxation of energy between different excitonic states but is consistent with a Förster-type transfer of energy between two chromophores with different absorption spectra. Assuming a similar structure as in the related C-phycocyanin [10], the transfer rate is within the range expected with the given distance and orientation between the chromophores and the calculated Förster radius [11]. In this work we also show that the APC trimers, especially those without 
the $8.9 \mathrm{kD}$ linker polypeptide, are rather unstable, even at $4^{\circ} \mathrm{C}$. In older samples, or samples without the linker polypeptide that show a shoulder about $620 \mathrm{~nm}$, a long (>10 ps) relaxation time appears which is probably related to the APC lifetimes reported previously [12-17]. A preliminary account of this work has been presented [18].

\section{Material and methods}

The APC trimers of Mastigocladus laminosus were isolated according to Füglistaller et al. [19]. The state of aggregation was determined by ultracentrifugation [20]. The trimeric preparations were kept frozen in $0.1 \mathrm{M}$ potassium phosphate buffer until used. Immediately after thawing the absorption spectrum of the trimer (sample No. 1) was very close to the spectrum of APC complex with a $8.9 \mathrm{kD}$ linker peptide $[15,121]$. The ratio of the absorption at the maximum $(653 \mathrm{~nm})$ to the absorption at the $620 \mathrm{~nm}$ shoulder was 2.0 (fig. 1). After keeping at $4^{\circ} \mathrm{C}$ for three weeks the spectrum became similar to the spectrum of linker-free allophycocyanin [15,21] with lower ratio of the absorption of $653 \mathrm{~nm}$ to the absorption at $620 \mathrm{~nm}$. This ratio was equal to 1.6. We have performed measurements with this "three

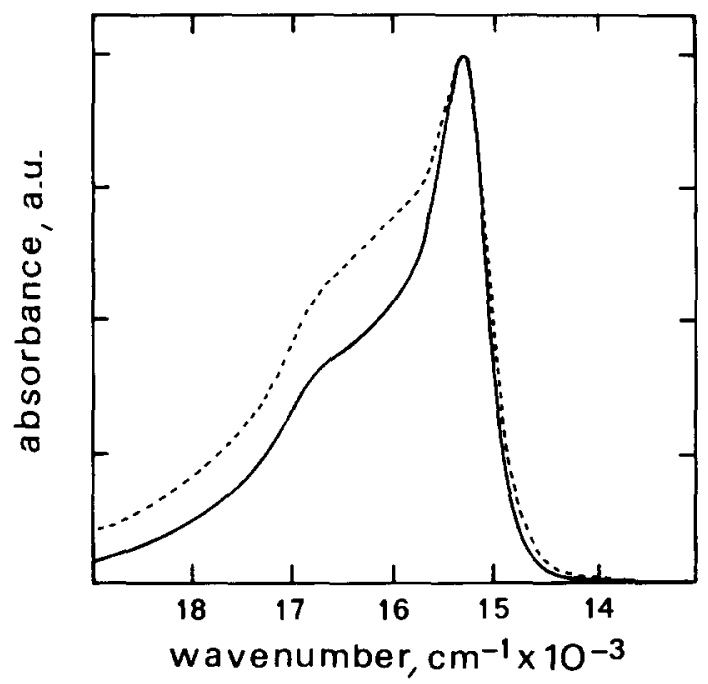

Fig. 1. Absorption spectra of different APC-trimer preparations, (-) sample No. 1, (--) sample No. 3. Amplitudes normalized to equal maximum absorbance. weeks" sample (sample No. 2) as well as with the "fresh" preparation. The APC monomers were obtained from sample No. 1 by adding KSCN to $1.2 \mathrm{M}$ directly before the measurements. We also studied a fresh trimeric preparation (sample No. 3 ) with ratio of absorption at $653 \mathrm{~nm}$ to absorption at 620 equal to 1.5 (fig. 1). This spectrum is similar to that of linker-free preparations in the literature $[15,21]$. No change of the maximum-to-shoulder ratio was observed after keeping sample No. 4 for three weeks at $4^{\circ} \mathrm{C}$.

All femtosecond absorption measurements were made at $20^{\circ} \mathrm{C}$ in a rotation cell of $1 \mathrm{~mm}$ optical path length. The absorbances of the samples at the excitation wavelength $(615 \mathrm{~nm})$ were 0.3 for APC sample No. 1, 0.5 for No. 2, 0.65 for No. 3 and 0.5 for APC monomers. $70 \mathrm{fs}$ pulses at $615 \mathrm{~nm}(\Delta \lambda=13 \mathrm{~nm})$ from a CPM laser were amplified at $10 \mathrm{kHz}$ repetition rate in a multipass jet amplifier [22]. These pulses served as both pump and probe pulses. Pumpprobe transient absorption measurements were performed with the polarization of the probe pulse set at $0^{\circ}, 54.7^{\circ}$ or $90^{\circ}$ to the polarization of the excitation pulse. Details of the detection system will be published later.

\section{Results}

Fig. 2 shows the photoinduced absorbance changes

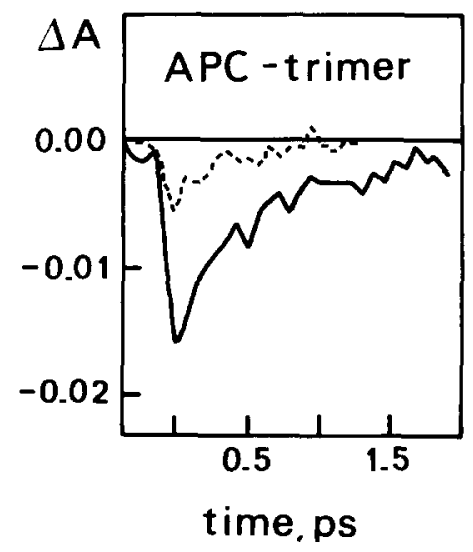

Fig. 2. Photo-induced absorbance changes in APC trimers (sample No. 1) at $615 \mathrm{~nm}$ with probe polarization parallel (-) and perpendicular (--) to the excitation polarization. 
in APC trimers (sample No. 1) at $615 \mathrm{~nm}$ immediately after thawing. The lifetime of the absorbance recovery kinetics is $0.5 \pm 0.1$ ps and the signal recovers approximately to the zero signal level. The anisotropy was found to be constant and equal to $0.4 \pm 0.1$. In the aged sample (sample No. 2) a fast initial signal was also observed with a recovery lifetime of $425 \pm 40 \mathrm{fs}$. In this sample, however, the fast signal did not recover to the zero level. At 2 ps after excitation about $20 \%$ of the signal still remained. Fig. 3 shows the results obtained with sample No. 3 (probably APC trimers without linker polypeptides). In this experiment excitation and probing pulses at $620 \mathrm{~nm}$ were used. In the semilogarithmic plot of the recovery kinetics measured with the probe pulse polarization parallel to the excitation pulse, single exponential kinetics with $\tau=440 \pm 3$ fs were
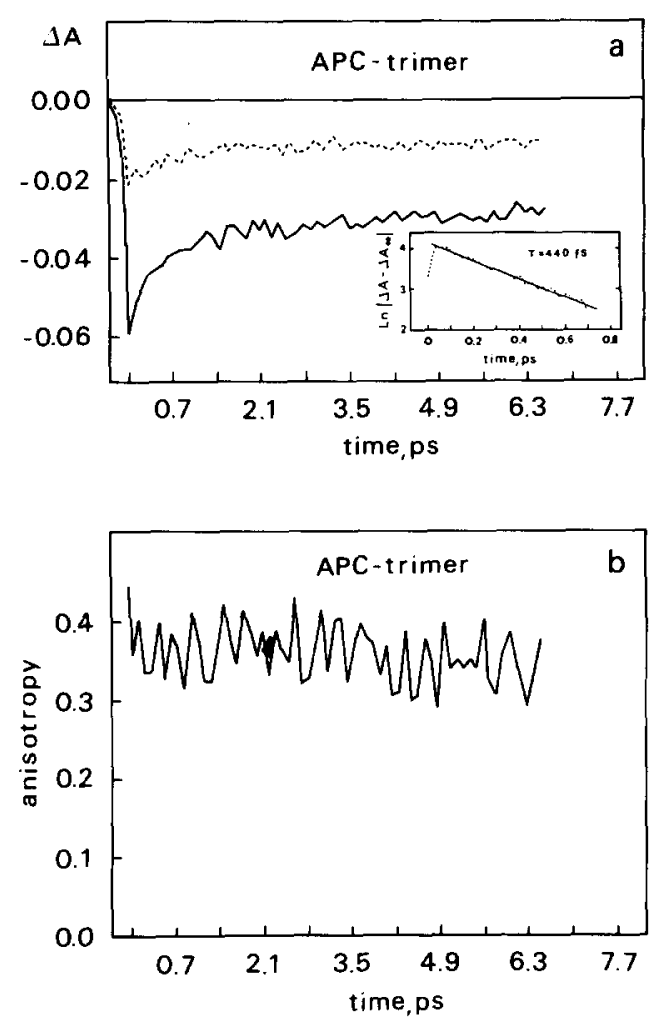

Fig. 3. (a) Time-resolved absorbance changes in APC trimers (sample No. 3) at $620 \mathrm{~nm}$ with probe light polarization parallel (-) and perpendicular (- - ) to the excitation light polarization. Inset: Semilogarithmic plot of the fast kinetics, probe light polarization parallel to excitation light polarization. (b) Time-resolved anisotropy of sample No. 3 . obtained. The anisotropy was constant and equal to $0.36 \pm 0.05$ within 6 ps after excitation (fig. $3 b$ ). In this sample the remaining of the bleaching measured 6 ps after excitation was approximately $50 \%$ of the initial bleaching at $t=0$. It cannot be excluded that kinetics with a lifetime longer than 10 ps partially account for the recovery in addition to the femtosecond one. Fig. 4 exhibits the absorbance changes measured with APC monomers. No evident decay kinetics were observed during the first 2 ps after excitation for the two polarizations of the probe pulses. The anisotropy was constant and equal to $0.42 \pm 0.05$. Summarizing our results with all three APC trimer samples we conclude, that (i) for all samples similar isotropic femtosecond kinetics were observed at 615$620 \mathrm{~nm}$ and (ii) anisotropy decay was not observed in any sample during the measurement period.

\section{Discussion}

We here discuss different explanations of the 0.5 ps kinetics observed in APC trimers. To do this it is necessary to return to the explanation of the large red-shift from 615 to $653 \mathrm{~nm}$ which occurs upon aggregation of monomers into trimers [15,21]. Two explanations of this red-shift have been suggested $[8,9]$. The most straightforward interpretation is that a conformational change occurs in the $\alpha$ - and/or $\beta$ chromophores as the $\alpha$ - and $\beta$-protein in different monomers bind to each other. the second and currently more popular explanation is that adjacent chromophores in the trimer interact strongly. As a consequence of this dipole-dipole interaction the monomer absorption band is split into two excitonic

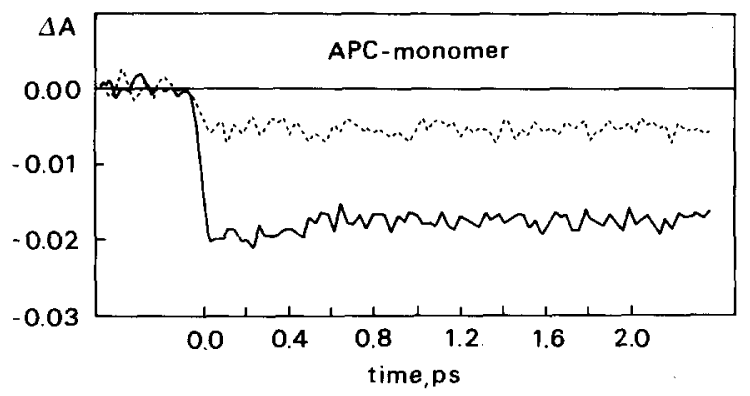

Fig. 4. Photo-induced absorbance changes in APC monomers at $615 \mathrm{~nm}$. Parallel (-) and perpendicular (--) polarization. 
states $[8,9]$. CD spectra have been analyzed with this model $[8,9,15]$, but it would probably also be possible to analyze the CD spectra as a sum of spectra from $\alpha$ and $\beta$ chromophores with different conformations without inferring excitonic interaction. Based on these models, we will discuss three possible explanations of the 0.5 ps absorbance recovery observed in APC trimers. These are; relaxation between two excitonic states, intramolecular vibrational relaxation and donor-acceptor energy transfer among spectrally different chromophores, respectively. In the first case we assume that we can excite one of the excitonic states selectively at $615 \mathrm{~nm}$. A relaxation to the lower state at $653 \mathrm{~nm}$ would not be seen as a fast ground-state recovery, however, since the excitationally coupled dimer will still be excited. If the power excited state has the same absorbance as the ground state at $615 \mathrm{~nm}$ would observe a fast absorption recovery as the energy relaxes from the upper to the lower excitonic state. In this case however, it is not expected that the polarization of this excited state transition (from the lower excited state) is parallel to the ground state transition at $615 \mathrm{~nm}$ as required by the high $(\approx 0.4)$ constant anisotropy. It is therefore very unlikely that our $0.5 \mathrm{ps}$ transient represents relaxation between two excitonic states. If the excitonic states are separated by just a few hundred $\mathrm{cm}^{-1}$, as suggested by the interpretation of the CD spectrum [15] and by comparison to the calculated interaction between $\alpha-84$ and $\beta-82$ chromophores in C-PC trimers [11] (which are presumably equivalent to the $\alpha-80$ and just $\beta-81$ ones in APC), our spectrally broad $\left(>100 \mathrm{fs} \mathrm{cm}^{-1}\right.$ ) pulses would just be able to excite a mixture of the two excitonic levels and inter-state relaxation would not be observed by us.

According to the second interpretation the $620 \mathrm{~nm}$ shoulder is due to a vibronic structure in both the $\alpha$ 80 and $\beta-81$ absorption bands. In this case the 0.5 ps kinetics could be interpreted as vibrational relaxation in the excited state. Again it seems highly unlikely, however, that at $615 \mathrm{~nm}$ the relaxed excited state absorption should be identical to that of the ground state. Furthermore no fast processes that might be ascribed to vibrational relaxation were observed in APC monomers or C-PC trimers and monomers.

It remains for us to discuss the interpretation of the $0.5 \mathrm{ps}$ kinetics in terms of donor-acceptor energy transfer. In this case we assume that the $\alpha$ - and $\beta$ chromophores have different conformations and thus also absorption spectra as a consequence of different local protein interactions. We further assume that the location and orientation of the chromophores are close to the situation in C-PC trimers [10]. For the fastest $\alpha-80 \leftrightarrow \beta-81$ transfer in C-PC trimers Sauer and Scheer [11] calculated a lifetime of $\approx 0.35 \mathrm{ps}$. The lifetime of $440 \mathrm{fs}$ measured in the APC trimers is thus in the expected range considering possible minor differences in the calculated Förster radius and in the crystallographic coordinates $[10,11]$. One further support of this model is that the anisotropy is constant, $\approx 0.4$, during the whole signal decay. The fact that the signal almost recovers to the base line in the more intact sample No. 1 supports our idea that the absorbance of the acceptor chromophore at $615 \mathrm{~nm}$ is small.

Samples showing a ratio of 650 to $620 \mathrm{~nm}$ absorbance which is only $\approx 1.5$ give rise to a high proportion of a long-lived ( $>10 \mathrm{ps}$ ) excited state. It was also found that only APC trimers with an absorbance ratio above 2 show a small $(>10 \%)$ contribution from long-lived states. It is however, not evident to us at the moment whether the $8.9 \mathrm{kD}$ linker polypeptide is absolutely required to get an "intact" spectrum or if the linker-free preparations simply are less stable under standard preparation and storing conditions. The long unresolved relaxation time observed clearly in samples 2 and 3 , but which might also be present to a smaller extent in sample 1 , is in our opinion due to a disruption of the donor-acceptor interaction (fig. 5) caused by (a) mono-trimer equilibrium or (b) beginning dissociation. One explanation of the change in the absorption spectrum, is that the acceptor chromophore (A) at $653 \mathrm{~nm}$ (fig. 1 ) in the "high 653 " form is shifted to $\approx 620 \mathrm{~nm}$ $\left(\mathrm{A}^{\prime}\right)^{\# 1}$. This might be due to a change in the interaction between the two APC monomers in the region of the $\alpha-80$ and $\beta-81$ binding sites as illustrated in fig. 5. As a consequence of this structural change two slow energy transfer channels will appear one in each of

$\#$ Whereas the donor-acceptor (D-A) distinction becomes meaningless at longer times and with spectrally more closely spaced chromophores [11] the conventional description seems to be appropriate here. 


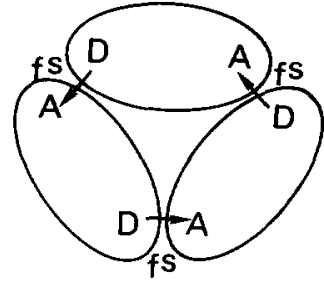

A

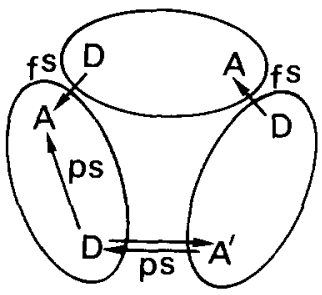

B

Fig. 5. A model for energy transfer pathways in intact APC trimers (A) and in partly disintegrated trimers (B). D is donor chromophore, $A$ is acceptor chromophore, $f \mathrm{~s}$ is subpicosecond transfer, ps is transfer with $\tau \geqslant 10$ ps.

the adjacent monomer units. The first most obvious channel being the transfer between $D$ and a modified acceptor, $A^{\prime}$. This model could well explain for some of the reported transfer times between 13 and $100 \mathrm{ps}$ different APC-trimer preparations [11-16]. The complete lack of fast ( $<1 \mathrm{ps}$ ) kinetics in APC monomers is of course in complete agreement with the donor-acceptor model and the monomer results will not be discussed further.

The anisotropy data also deserve some comment, since our results are in disagreement with earlier picosecond absorption measurements [12]. One explanation for differing anisotropies extrapolated to time $t=0(r(0))$ might be a contribution to the absorption from the excited state of the acceptor, which may have a different polarization than the ground state. This could also explain the relatively low anisotropy $(r(0)) \approx 0.2$ ) observed at $648 \mathrm{~nm}[11]$. The same might be true for shorter wavelengths, i.e. $\lambda=595 \mathrm{~nm}$, used in the work of Back and Sauer [16], where $r(0)$ was estimated to be 0.28 . In general one has to be aware, however, that anisotropy measurements usually involve larger errors than isotropic kinetics. This is due partly to instrumental factors and partly to the fact that a difference between two signals is measured. The high anisotropy observed for APC monomers is close to the theoretically expected $r=0.4$, which lends support to the anisotropy measured on APC trimers in this work. In spite of this discrepancy in the literature it is clear from our work in APC trimers that the isotropic 0.5 ps decay is not followed by an equally fast anisotropic relaxation in APC trimers.

\section{Acknowledgement}

We thank the Swedish Natural Science Research Council, the Royal Swedish Academy of Sciences, the Russian Academy of Sciences and the Deutsche Forschungsgemeinschaft (RF and HS in SFB 143, project A1) for financial support. Mrs. Eva Vikström is acknowledged for preparing the figures and Mrs. Anita Öystilä for typing this manuscript.

\section{References}

[1] A.N. Glazer, Biochim. Biophys. Acta 768 (1984) 29.

[2] A.R. Holzwarth, Topics Photosynth. 8 (1987) 95.

[3] S.S. Brody, G. Porter, C.J. Tredwell and J. Barber, Photobiochem. Photobiophys. 2 (1981) 11.

[4] M. Mimuro, I. Yamazaki, N. Tamai and Katoh, Biochim. Biophys. Acta 973 (1989) 153.

[5] A.S. Davydov, Soviet Phys. Uspekhi 82 (1964) 145.

[6] Th. Förster, Ann. Physik 26 (1948) 55.

[7] W. Sidler, J. Gysi, E. Isker and H. Zuber, Z. Physiol. Chem. 362 (1981) 611 .

[7] K. Csatorday, D. Guard-Friar, R. MacColl and D.S. Berns, Photochem. Photobiol. 47 (1988) 285.

[9] O. Canaani and E. Gantt, Biochemistry 19 (1988) 157.

[10] T. Schirmer, W. Bode and R. Huber, J. Mol. Biol. 196 (1987) 677.

[11] K. Sauer and H. Scheer, Biochim. Biophys. Acta 936 (1988) 157.

[12] T. Gillbro, A. Sandström, V. Sundström, R. Fischer and H. Scheer, in: Photosynthetic light-harvesting systems, eds. $\mathrm{H}$. Scheer and S. Scheider (Walter de Gruyter, Berlin, 1988) p. 457.

[13] P. Maxson, K. Sauer and A.N. Glazer, in: Photosynthetic light-harvesting systems, eds. H. Scheer and S. Scheider (Walter de Gruyter, Berlin, 1988) p. 439.

[14] E. Bittersmann, W. Reuter, W. Wehrmeyer and A.R. Holzwarth, in: Photosynthetic light-harvesting systems, eds. H. Scheer and S. Scheider (Walter de Gruyter, Berlin, 1988) p. 451.

[15] A.R. Holzwarth, E. Bittersmann, W. Reuter and W. Wehrmeyer, Biophys. J. 57 (1990) 133.

[16] W.F. Beck and K. Sauer, Biophys. J. 59 (1991) 32(A). 
[17] S.W. Yeh, A.N. Glazer and J.H. Clark, J. Phys. Chem. 90 (1986) 4578.

[18] E.V. Khoroshilov, I.V. Kryukov, P.G. Kryukov, A.V. Sharkov and T. Gillbro, SPIE Vol. 1403, Laser applications in life sciences (1990) p. 431.

[19] P. Füglistaller, H. Widmer, W. Sidler, G. Frank and H. Zuber, Arch. Microbiol. 129 (1981) 268.

[20] R.G. Martin and B.N. Ames, J. Biol. Chem. 236 (1961) 1374.
[21] P. Füglistaller, M. Mimuro, F. Suter and H. Zuber, Biol. Chem. 368 (1987) 353.

[22] E.V. Khoroshilov, I.V. Kryukov, P.G. Kryukov and A.V. Sharkov, in: Ultrafast phenomena VI, eds. T. Yajima, K. Yoshihara, C.B. Harris and S. Shionoya (Springer, Berlin, $1988)$ p. 22. 